\title{
Viable periodic solutions in state-dependent impulsive problems
}

\author{
Grzegorz Gabor
}

Received: 23 January 2014 / Accepted: 17 February 2015 / Published online: 3 March 2015

(C) The Author(s) 2015. This article is published with open access at Springerlink.com

\begin{abstract}
Periodic solutions of state-dependent impulsive ODEs in a prescribed set of constraints are examined. The so-called impulsive index is introduced, as a topological tool, and its properties are studied. Some sufficient conditions for its homotopy property are discussed in detail. In a construction of the impulsive index the fixed point index on ANRs is applied to an induced discrete semidynamical system on a barrier where jumps occur. Several illustrative examples are added.
\end{abstract}

Keywords Periodic solution - Viable solution - Fixed point index · State-dependent impulsive problems $\cdot$ Impulse function

Mathematics Subject Classification Primary 34B37; Secondary 34C25 - 37B30 . $47 \mathrm{H} 10 \cdot 47 \mathrm{H} 11$

\section{Introduction}

Impulsive ordinary, or partial, differential equations became a strongly growing field of research in a last half of a century. Some results in this field are simple modifications and generalizations of analogous results for non-impulsive problems but, beyond them, there are essentially new, nontrivial and interesting questions requiring new methods and proof arguments. We meet this especially in state-dependent impulsive problems where jumps occur in variable times. A study in this direction was initiated by Mil'man and Myshkis in [18]. Problems with impulses in variable times are met in e.g. physical and biological models (cf. [3]), where concerned quantities rapidly change when they attain some barriers or some critical levels in a phase space or in an extended phase space. In an analysis of solutions of such problems, in contrast to those with fixed impulse times, one also has to

G. Gabor $(\bowtie)$

Faculty of Mathematics and Computer Science, Nicolaus Copernicus University,

Chopina 12/18, 87-100 Toruń, Poland

e-mail: ggabor@mat.umk.pl 
take into account a geometry or topology of barriers and their relationships with dynamics of the problem. This area is still poorly investigated, especially in the context of applications of known or construction of new appropriate topological tools.

In the paper we focus our attention on detecting a periodic behavior of some of trajectories for state-dependent impulsive ODEs under an additional requirement that these trajectories are viable in a prescribed closed subset $K \subset \mathbb{R}^{n}$ of a state space. In other words, we are going to find a solution to the following periodic problem

$$
\begin{cases}\dot{x}(t)=f(x(t)) & \text { for } \text { a.e. } t \geq 0, x \in \mathbb{R}^{n}, \\ x(t) \in K, & \\ x\left(t^{+}\right):=\lim _{s \rightarrow t^{+}} x(s)=I(x(t)) & \text { for } x(t) \in M \subset \partial K, \\ x(0)=x\left(T_{x}\right), & \text { for } \operatorname{some}_{x}>0,\end{cases}
$$

where $f: \mathbb{R}^{n} \supset \Omega \rightarrow \mathbb{R}^{n}$ is sufficiently smooth to generate a semiflow, and $I: M \rightarrow \mathbb{R}^{n}$ is a continuous impulse function. More specific assumptions on $f, M$ and $I$ will be given later. The notion of viability used above is motivated by biological models where some species survives only if it does not leave a region of safety $K$ given as a set of constraints.

There are only a few papers concerning problem (1.1) (see, e.g. [8,9,14,17]). In most of them additional Nagumo-type tangency conditions are assumed to ensure that from every point of $K$ there starts at least one trajectory, even with impulses taken into account, remaining in $K$ for some nontrivial time interval. Then the fixed point theory can be applied for a suitably defined Poincaré-Krasnoselskii operator.

If tangency conditions do not hold on the whole boundary, we allow trajectories for a non-impulsive problem, even all of them, to leave $K$ through a so-called exit set $K^{-}$. Of course, we want to prevent this escape, so we place a barrier $M$ in $K^{-}$trying to return at least some of them to the set $K$ via the impulse function $I$ (see [14]). In some biological models it means an external impact (e.g. restocking the lake). In [14] the impulse function was assumed to satisfy $I(M) \subset K$. Analysis of models in mathematical biology shows that it is worth considering a weaker assumption $I(M) \subset \mathbb{R}^{n}$, in practice at least $I(M) \cap K \neq \emptyset$. This situation is more complicated and needs deeper local topological tools to study problem (1.1). In the paper we construct a so-called impulsive index, which is a fixed point index of suitably defined continuous map in an exit set $K^{-}$. Its nontriviality will imply the existence of viable periodic trajectories in $K$.

The paper is organized as follows. In Sect. 2 at first we give basic assumptions, and define the impulsive index. Then properties of the index are presented, discussed and proved. Several examples are given to illustrate the results. In Sect. 3 we present geometric sufficient conditions for a homotopy property of the index with some results, concerning exit sets, interesting in themselves. The Poincaré map technique is adapted to compute the impulsive index in a neighborhood of a periodic point in Sect. 4 (see also [14] where this technique was proposed). The last section (Sect. 5) contains several concluding remarks concerning an alternative construction of the impulsive index. Advantages and disadvantages of this alternative technique are discussed.

\section{Impulsive index}

Let $K \subset \mathbb{R}^{n}$ be an arbitrary closed subset, $\Omega \supset K$ an open neighborhood in $\mathbb{R}^{n}$, and $f: \Omega \rightarrow \mathbb{R}^{n}$ be a sufficiently smooth vector field such that the equation $\dot{x}=f(x)$ generates a semiflow $\pi$ on $\Omega$. We are interested in viable in $K$ solutions so, without any loss of generality, we can assume in the sequel that $\Omega=\mathbb{R}^{n}$. Let us recall that by a solution of problem (1.1) we 
mean a left-continuous function $x:[0, \infty) \rightarrow \mathbb{R}^{n}$, with a discrete set of discontinuity points, absolutely continuous between these points, and satisfying $\dot{x}(t)=f(x(t))$ in a.e. $t \geq 0$, and $x\left(t^{+}\right)=I(x(t))$ in discontinuity points. The set of functions from $[0, \infty)$ to $\mathbb{R}^{n}$ which are continuous between discontinuity points taken from a discrete set, and left-continuous in these points will be denoted below by $P C\left([0, \infty), \mathbb{R}^{n}\right)$.

In each point $x \in K$ we can consider the Bouligand tangent cone

$$
T_{K}(x):=\left\{v \in \mathbb{R}^{n} \mid \liminf _{h \rightarrow 0^{+}} \operatorname{dist}(x+h v, K) / h=0\right\} .
$$

Obviously, if the following tangency condition

$$
f(x) \in T_{K}(x) \text { for every } x \in K
$$

is satisfied, then all solutions starting in $K$ remain in this set forever (see the first results of this type in [19] and [6]). If it is not satisfied, the following exit set for $K$

$$
K^{-}=K^{-}(f):=\left\{x_{0} \in K \mid \forall \varepsilon>0: \pi\left(\left\{x_{0}\right\} \times(0, \varepsilon)\right) \not \subset K\right\}
$$

appears. If topological properties of the exit set sufficiently differs from properties of $K$, then still there exists at least one viable trajectory in $K$. This was firstly proved in the celebrated Ważewski paper [20]. More precisely, if $K^{-}$is closed and it is not a strong deformation retract of $K$, then a viable solution exists (comp. [11]). In the paper we are interested in even worse case:

All absolutely continuous solutions to $\dot{x}=f(x)$ leave the set $K$.

It means, in consequence, that $K$ is strongly deformed via homotopy onto $K^{-}$. Namely, the exit function $\tau_{K}: K \rightarrow[0, \infty), \tau_{K}\left(x_{0}\right):=\sup \left\{t \geq 0 \mid \pi\left(\left\{x_{0}\right\} \times[0, t]\right) \subset K\right.$ is continuous, and we can define a homotopy $h: K \times[0,1] \rightarrow K, h\left(x_{0}, \lambda\right):=\pi\left(x_{0}, \lambda \tau_{K}\left(x_{0}\right)\right)$. Putting $r\left(x_{0}\right):=h\left(x_{0}, 1\right)$ we obtain a (continuous) retraction $r: K \rightarrow K^{-}$.

Hence, in the whole paper we assume that

$$
h(x, \alpha):=\pi\left(x, \alpha \tau_{K}(x)\right), x \in K, \text { is a deformation of } K \text { onto } K^{-} .
$$

Of course, (2.2) implies that $K^{-}$is a closed subset of $K$. When we consider a family $\pi_{\lambda}$ of semiflows, we will assume, analogously, that deformations $h_{\lambda}$ are given, see below.

Let $M \subset K^{-}$be an impulse set, i.e., there is an impulse function $I: M \rightarrow \mathbb{R}^{n}$ moving instantly each trajectory reaching the set $M$ in a point $p \in M$ to a point $I(p) \in \mathbb{R}^{n}$. In practice, we will be interested in impulse functions satisfying $I(M) \cap K \neq \emptyset$ which means that some trajectories are moving back to $K$. In our considerations we assume that

(A1) $K^{-}$is a closed ANR,

(A2) $M=c l_{K^{-}}\left(\right.$int $\left._{K^{-}} M\right)$,

(A3) $I$ is a compact map, i.e. $I(M)$ is relatively compact,

(A4) $\operatorname{dist}(I(M), M)>0$,

where $i n t_{K^{-}} A, c l_{K^{-}} A$ and, in the sequel, $\partial_{K^{-}} A$ denote the relative interior, closure and boundary of a set $A \subset K^{-}$in $K^{-}$, respectively. Note that, if $M$ is compact, which is often the case, (A4) is implied by $I(M) \cap M=\emptyset$. This condition or (A4) is assumed in many papers concerning impulsive semidynamical systems (see, e.g., [1,5, 10]). It implies, in particular, that for every $x_{0} \in K \backslash M$ the sequence $\sigma_{0}\left(x_{0}\right)=0, \sigma_{1}\left(x_{0}\right)=\phi\left(x_{0}\right), \sigma_{m+1}\left(x_{0}\right)=$ $\sigma_{m}\left(x_{0}\right)+\phi\left((I \circ r)^{m}\left(x_{0}\right)\right)$ satisfies the condition $\lim _{m \rightarrow \infty} \sigma_{m}\left(x_{0}\right)=\infty$, where $\phi(x)=$ $\inf \{s>0 \mid \pi(\{x\} \times[0, s)) \cap M=\emptyset$ and $\pi(x, s) \in M\}$. 
We define $M^{I}:=I^{-1}(K)$ and

$$
g: M^{I} \rightarrow K^{-}, g(x):=r(I(x)) \text { for } x \in M^{I} .
$$

Denote

$$
\mathcal{P}:=\left\{x \in P C\left([0, \infty), \mathbb{R}^{n}\right) \mid x \text { is a viable periodic solution to }(1.1)\right\} .
$$

Notice that, since every absolutely continuous solution to $\dot{x}=f(x)$ leaves the set $K$, there is a correspondence between elements of $\mathcal{P}$ and fixed points of iterations of the map $g$ defined in (2.3). Indeed, let $x \in \mathcal{P}$ start from $x_{0} \in K$. Then $z=\pi\left(x_{0}, \tau_{K}\left(x_{0}\right)\right) \in M$, and there is a minimal $m \in \mathbb{N}$ such that $x_{0}=\pi\left(I\left(g^{m}(z)\right), s\right)$ for some $s \in\left[0, \tau_{K}\left(I\left(g^{m}(z)\right)\right)\right]$. It implies that $z=\pi\left(x_{0}, \tau_{K}\left(x_{0}\right)\right)=g^{m+1}(z)$, so $z$ is a fixed point of $g^{m+1}$. On the other hand, if $z=g^{m}(z)$, then the map $x(0):=z, x(t):=\pi\left(I\left(g^{k}(z)\right), t-\sigma_{k}(I(z))\right)$ for $t \in$ $\left(\sigma_{k}(I(z)), \sigma_{k+1}(I(z))\right], k \geq 0$, is a viable periodic solution to problem (2.2) starting from $z$.

In particular, each fixed point of $g$ generates a periodic viable trajectory in $K$. This motivates us to construct a fixed point index type topological tool detecting fixed points of $g$.

Let $V$ be an arbitrary open subset of $K^{-}$. Let us define

$$
U_{V}:=\operatorname{int}_{K^{-}}\left(I^{-1}(K) \cap V\right) \subset V,
$$

and assume that

$$
\text { Fix } g \cap U_{V} \text { is a compact set. }
$$

We define an impulsive index of $\pi$ on $V$ as

$$
\operatorname{ind}_{K}(\pi, I, V):=\operatorname{ind}\left(g, U_{V}\right),
$$

where ind $\left(g, U_{V}\right)$ is a fixed point index for compact maps on ANRs (see [16]).

Below we show the following main properties of the impulsive index.

Theorem 2.1 (Existence) If ind in $_{(}(\pi, I, V) \neq 0$, then there exists a viable periodic solution to $(1.1)$ reaching the set $V$.

Theorem 2.2 (Additivity) Let $K^{-} \supset V=V_{1} \cup V_{2}, V_{i}$ open and $V_{1} \cap V_{2}=\emptyset$. If Fix $g \cap\left(U_{V}\right)$ is a compact set, then

$$
\operatorname{ind}_{K}(\pi, I, V)=\operatorname{ind}_{K}\left(\pi, I, V_{1}\right)+\operatorname{ind}_{K}\left(\pi, I, V_{2}\right) .
$$

Theorem 2.3 (Homotopy I) Let $\pi_{\lambda}, \lambda \in[0,1]$, be a continuous family of semiflows, i.e. the map $\mathbb{R}^{n} \times[0, \infty) \times[0,1] \ni(x, t, \lambda) \mapsto \pi_{\lambda}(x, t)$ is continuous. Assume that, for every $\lambda \in[0,1]$, the exit set $K_{\lambda}^{-}$for $\pi_{\lambda}$ is a closed ANR, there are retractions $r_{\lambda}: K \rightarrow K_{\lambda}^{-}$ along trajectories, i.e., $r_{\lambda}(x)=\pi_{\lambda}\left(x, \tau_{K}(x)\right)$, and $M \subset K_{\lambda}^{-}$satisfies $M=c l_{K_{\lambda}^{-}}\left(\right.$int $\left._{K_{\lambda}^{-}} M\right)$. Moreover, assume that $X=\bigcup_{\lambda \in[0,1]} K_{\lambda}^{-} \in A N R$, and the set $\bigcup_{\lambda \in[0,1]} r_{\lambda}(I(M) \cap K)$ is relatively compact in $X$.

Let $V \subset \bigcap_{\lambda \in[0,1]} K_{\lambda}^{-}$be open in every $K_{\lambda}^{-}$, and such that $U_{V_{\lambda_{0}}}$ is an open subset of $X$ for some $\lambda_{0} \in[0,1]$, and Fix $g_{\lambda} \cap U_{V_{\lambda}}$ is compact, where $g_{\lambda}:=r_{\lambda} \circ I: M^{I} \rightarrow K_{\lambda}^{-}$.

Then the numbers ind ${ }_{K}\left(\pi_{\lambda}, I, V\right)$ are well defined, and

$$
\operatorname{ind}_{K}\left(\pi_{0}, I, V\right)=\operatorname{ind}_{K}\left(\pi_{1}, I, V\right) .
$$

Theorem 2.4 (Homotopy II) Let $\pi_{\lambda}, \lambda \in[0,1]$, be a continuous family of semiflows, and assume that, for every $\lambda \in[0,1]$, the exit set $K_{\lambda}^{-}$for $\pi_{\lambda}$ is a closed ANR, there are retractions $r_{\lambda}: K \rightarrow K_{\lambda}^{-}$along trajectories, impulse sets $M_{\lambda} \subset K_{\lambda}^{-}$satisfying $M_{\lambda}=c l_{K_{\lambda}^{-}}\left(\right.$int $\left._{K_{\lambda}^{-}} M_{\lambda}\right)$, 
Fig. 1 A system with $V \cap M=\emptyset$

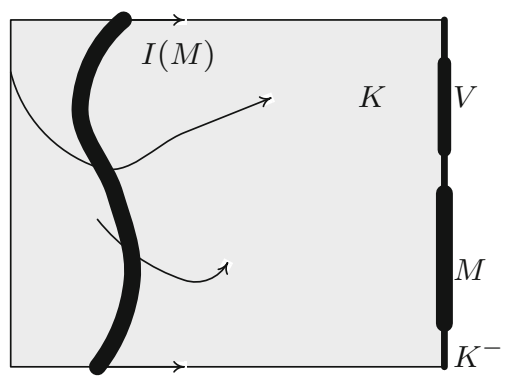

Fig. 2 A system with $I(M) \cap K=\emptyset$

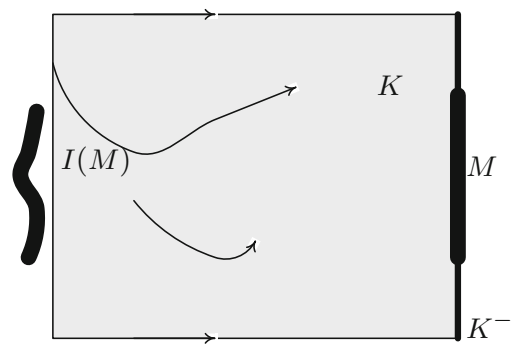

and impulse functions $I_{\lambda} \rightarrow \mathbb{R}^{n}$. Moreover, assume that, for every pair $0 \leq a \leq b \leq 1$, the set $X_{a}^{b}=\bigcup_{\lambda \in[a, b]} K_{\lambda}^{-}$is an ANR, and the set $\bigcup_{\lambda \in[a, b]} r_{\lambda}\left(I_{\lambda}\left(M_{\lambda}\right) \cap K\right)$ is relatively compact in $X_{a}^{b}$.

Let $V_{\lambda} \subset K_{\lambda}^{-}$be such that $\bigcup_{\lambda \in[0,1]} U_{V_{\lambda}} \times\{\lambda\}$ is an open subset of $X_{0}^{1}$, and such that $F:=\bigcup_{\lambda \in[0,1]}\left(\left(\right.\right.$ Fix $\left.\left.g_{\lambda} \cap U_{V_{\lambda}}\right) \times\{\lambda\}\right)$ is compact, where $g_{\lambda}:=r_{\lambda} \circ I_{\lambda}: M_{\lambda}^{I} \rightarrow K_{\lambda}^{-}$and $M_{\lambda}^{I}:=I_{\lambda}^{-1}\left(I_{\lambda}\left(M_{\lambda}\right) \cap K\right)$. Then

$$
\operatorname{ind}_{K}\left(\pi_{0}, I_{0}, V_{0}\right)=\operatorname{ind}_{K}\left(\pi_{1}, I_{1}, V_{1}\right) .
$$

Before proofs, let us comment some of assumptions in the above theorems.

Remark 2.5 If Fix $g \cap \partial_{K^{-}}\left(U_{V}\right)=\varnothing$ in (2.5) and Theorem 2.1, then Fix $g \cap U_{V}$ is a compact set. Indeed, it follows from the continuity of $g$ and compactness of the impulse map in the following way. We have $I\left(M^{I}\right) \subset K, K$ is closed in $\mathbb{R}^{n}$, and $c l(I(M))$ is compact. Hence, $\operatorname{cl}\left(I\left(M^{I}\right)\right) \subset K$ is also compact. Now, $\operatorname{cl}\left(r\left(I\left(M^{I}\right)\right)\right) \subset \operatorname{cl}\left(r\left(\operatorname{cl}\left(I\left(M^{I}\right)\right)\right)\right)=$ $r\left(\operatorname{cl}\left(I\left(M^{I}\right)\right)\right) \subset K^{-}$, and $r\left(\operatorname{cl}\left(I\left(M^{I}\right)\right)\right)$ is compact because $r$ is continuous.

If $I(V \cap M) \subset K$ in Theorem 2.1, then $U_{V}=i n t_{K^{-}}\left(I^{-1}(K \cap V)=i n t_{K^{-}}(V \cap M)\right.$, and

$$
\operatorname{ind}_{K}(\pi, I, V)=\operatorname{ind}\left(g, i n t_{K^{-}}(V \cap M)\right) .
$$

In particular, if $V \subset M$ is an open subset of $K^{-}$, then

$$
\operatorname{ind}_{K}(\pi, I, V)=\operatorname{ind}(g, V) \text {, }
$$

as in paper [14].

If $V \cap M=\emptyset$ (see Fig. 1) or $I(M) \cap K=\emptyset$ (see Fig. 2), then $U_{V}=\emptyset$, and we trivially obtain $\operatorname{ind}_{K}(\pi, I, V)=0$.

The assumption $M \subset \bigcap_{\lambda \in[0,1]} K_{\lambda}^{-}$in Theorem 2.3 is sensible. One knows that

$$
K_{\Rightarrow}^{\lambda}:=\left\{x \in \partial K ; f_{\lambda}(x) \notin T_{K}(x)\right\} \subset K_{\lambda}^{-} \subset \operatorname{cl} K_{\Rightarrow}^{\lambda},
$$


if semiflows are generated by equations $\dot{x}=f_{\lambda}(x)$ ([7], Lemma 5.1). Assume that $M \subset$ $\left\{x \in \partial K ; f_{\lambda_{0}}(x) \notin T_{K}(x)\right\}$ and $\left(f_{\lambda}\right)$ is a continuous family of maps. Then, for $\lambda$ sufficiently near $\lambda_{0}, M \subset\left\{x \in \partial K ; f_{\lambda}(x) \notin T_{K}(x)\right\} \subset K_{\lambda}^{-}$.

The existence of a family of retractions $r_{\lambda}$ in Theorem 2.3 is also natural. Indeed, if $K$ is bounded and $\pi_{\lambda_{0}}$ does not have a viable trajectory in $K$ for some $\lambda_{0} \in[0,1]$, then $\pi_{\lambda}$ does not have a viable trajectory in $K$ for $\lambda$ sufficiently close to $\lambda_{0}$. To check this, let us assume, by contrary, that there is a sequence $\lambda_{n} \rightarrow \lambda_{0}$ and a viable trajectory $\pi_{\lambda_{n}}\left(\cdot, x_{n}\right)$ in $K$ for every $n \geq 1$. By the compactness of $K$ we can assume, without any loss of generality, that $x_{n} \rightarrow x \in K$. From the continuity of a family of semiflows it follows that $\pi_{\lambda_{0}}(\cdot, x)$ is viable in $K$; a contradiction.

Observe that in Theorem 2.4 we have a family of maps $g_{\lambda}$ with possibly different domains and codomains, and, in fact, we consider an otopy introduced in [4] in the context of vector fields on manifolds.

Proof of Theorem 2.1. It is sufficient to notice that $0 \neq \operatorname{ind}_{K}(\pi, I, V)=\operatorname{ind}\left(g, U_{V}\right)$ implies Fix $g \cap U_{V} \neq \emptyset$ so, there is a point $p \in U_{V} \subset V$ with $r(I(p))=p$. This means that a solution of (1.1) starting from $I(p)$ is viable in $K$ and periodic, and touches the barrier in $p \in V$.

Proof of Theorem 2.2. Notice that

$$
\begin{aligned}
U_{V} & =i n t_{K^{-}}\left(I^{-1}(K) \cap V\right) \\
& =i n t_{K^{-}}\left(I^{-1}(K) \cap\left(V_{1} \cup V_{2}\right)\right) \\
& =i n t_{K^{-}}\left(\left[I^{-1}(K) \cap V_{1}\right] \cup\left[I^{-1}(K) \cap V_{2}\right]\right.
\end{aligned}
$$

Denote $A:=I^{-1}(K) \cap V_{1}$ and $B=I^{-1}(K) \cap V_{2}$. We are going to check that $U_{V}=U_{V_{1}} \cup U_{V_{2}}$, i.e., $i n t_{K^{-}}(A \cup B)=i n t_{K^{-}} A \cup i n t_{K^{-}} B$. Of course, ' $\supset$ ' is always true. It is sufficient to prove that each open set contained in $A \cup B$ can be represented as a sum of open subsets in $A$ and $B$, respectively.

Let $W$ be an open subset of $K^{-}$contained in $A \cup B=I^{-1}(K) \cap V$. Define $W_{1}:=W \cap A$ and $W_{2}:=W \cap B$. Since $W_{1} \subset V_{1}$ and $V_{1}$ is open in $K^{-}$, for an arbitrary $x \in W_{1}$ there exists $r_{0}>0$ such that $B\left(x, r_{0}\right) \subset V_{1}$, where $B\left(x, r_{0}\right)$ is an open ball in $K^{-}$. The set $W$ is open so there is $0<r \leq r_{0}$ such that $B(x, r) \subset W \subset I^{-1}(K) \cap V$. Therefore, $B(x, r) \subset V_{1} \cap I^{-1}(K)=A$. Similarly we check that $W_{2}$ is an open set contained in $B$.

Now, from the assumption it follows that Fix $g \cap\left(U_{V_{1}} \cup U_{V_{2}}\right)$ is a compact set. Moreover, $U_{V_{1}} \cup U_{V_{2}}=\emptyset$ so Fix $g \cap U_{V_{i}}$ is also compact for $i=1,2$. By the additivity property of the fixed point index on ANRs we obtain

$$
\begin{aligned}
\operatorname{ind}_{K}(\pi, I, V) & =\operatorname{ind}\left(g, U_{V}\right)=\operatorname{ind}\left(g, U_{V_{1}}\right)+\operatorname{ind}\left(g, U_{V_{2}}\right) \\
& =\operatorname{ind}_{K}\left(\pi, I, V_{1}\right)+\operatorname{ind}_{K}\left(\pi, I, V_{2}\right) .
\end{aligned}
$$

Proof of Theorem 2.3. At first we prove that $U_{V_{\lambda}}=U_{V_{\lambda_{0}}}$ for every $\lambda \in[0,1]$. To do this we take any open set $W \subset K_{\lambda}^{-}$contained in $I^{-1}(K) \cap V$. Let $\lambda^{\prime} \in[0,1]$ be arbitrary. Since $V$ is open in $K_{\lambda}^{-}$and in $K_{\lambda^{\prime}}^{-}$, there is $r_{0}>0$ such that for every $0<r \leq r_{0}$ we have $B_{K_{\lambda}^{-}}(x, r) \cup B_{K_{\lambda^{\prime}}^{-}}(x, r) \subset V$, and consequently, $B_{V}(x, r)=B_{K_{\lambda}^{-}}(x, r)=B_{K_{\lambda^{\prime}}^{-}}(x, r)$, where these balls are in spaces $V, K_{\lambda}^{-}, K_{\lambda^{\prime}}^{-}$, respectively. But $W$ is open in $K_{\lambda}^{-}$so there is $0<\delta \leq r_{0}$ such that $B_{K_{\lambda}^{-}}(x, \delta) \subset W$ which implies that $B_{K_{\lambda^{\prime}}^{-}}(x, \delta)=B_{K_{\lambda}^{-}}(x, \delta) \subset W$. Hence, $W$ is open in $K_{\lambda^{\prime}}^{-}$. 
Consider the maps $g_{\lambda}$ as $g_{\lambda}: M^{I} \rightarrow X$, i.e., with a codomain $X$. By the assumption, the set $U_{V_{\lambda_{0}}}$ is open in $X$. Therefore, we have a homotopy $g_{\lambda}: U_{V_{\lambda_{0}}} \rightarrow X$. Now, we use the contraction and homotopy properties of the fixed point index on ANRs to obtain

$$
\begin{aligned}
\operatorname{ind}_{K}\left(\pi_{0}, I, V\right) & =\operatorname{ind}\left(g_{0}, U_{V_{\lambda_{0}}}\right)=\operatorname{ind}_{X}\left(g_{0}, U_{V_{\lambda_{0}}}\right) \\
& =\operatorname{ind}_{X}\left(g_{1}, U_{V_{\lambda_{0}}}\right)=\operatorname{ind}_{K}\left(\pi_{1}, I, V\right),
\end{aligned}
$$

where, to distinguish, $\operatorname{ind}_{X}$ stands for the fixed point index on the ANR $X$.

Remark 2.6 It is not obvious that $U_{V_{\lambda_{0}}}$, which is open in every $K_{\lambda}^{-}$, is open in $X$, simultaneously. Some sufficient conditions for this property are presented in a separate section (see Sect. 3).

Proof of Theorem 2.4. We show that the index $\operatorname{ind}_{K}\left(\pi_{\lambda}, I_{\lambda}, V_{\lambda}\right)$ is locally constant, i.e., for every $\lambda_{0} \in[0,1]$ there exists $\delta>0$ such that $\operatorname{ind}_{K}\left(\pi_{\lambda}, I_{\lambda}, V_{\lambda}\right)=\operatorname{ind}_{K}\left(\pi_{\lambda_{0}}, I_{\lambda_{0}}, V_{\lambda_{0}}\right)$ for each $\lambda \in\left(\lambda_{0}-\delta, \lambda_{0}+\delta\right) \cap[0,1]$.

Fix any $\lambda_{0} \in[0,1]$. From the assumption on the set $F$ it follows that $F_{\lambda_{0}}:=$ Fix $g_{\lambda_{0}} \cap U_{V_{\lambda_{0}}}$ is a compact set as an image of $F$ by the projection $p: \partial K \times[0,1] \rightarrow \partial K, p(x, \lambda)=x$. For every point $x \in F_{\lambda_{0}}$ there exists $r(x)>0$ such that $B_{X}(x, r(x)) \times\left(\lambda_{0}-r(x), \lambda_{0}+r(x)\right) \subset$ $\bigcup_{\lambda \in[0,1]} U_{V_{\lambda}} \times\{\lambda\}$. Hence, we can take a finite covering $\left\{B_{X}\left(x_{k}, r\left(x_{k}\right)\right) \times\left(\lambda_{0}-r\left(x_{k}\right), \lambda_{0}+\right.\right.$ $\left.\left.r\left(x_{k}\right)\right)\right\}_{k=1}^{m}$ of $F_{\lambda_{0}} \times\left\{\lambda_{0}\right\}$, and its Lebesgue number $l$. Then, denoting $I_{\lambda_{0}}:=\left(\lambda_{0}-l, \lambda_{0}+\right.$ l) $\cap[0,1]$, we obtain

$$
F_{\lambda_{0}} \times\left\{\lambda_{0}\right\} \subset \bigcup_{x \in F_{\lambda_{0}}}\left(B_{X}(x, l) \times I_{\lambda_{0}}\right)=\left(\bigcup_{x \in F_{\lambda_{0}}} B_{X}(x, l)\right) \times I_{\lambda_{0}} \subset \bigcup_{\lambda \in I_{\lambda_{0}}}\left(U_{V_{\lambda}} \times\{\lambda\}\right) .
$$

Denote $U:=\bigcup_{x \in F_{\lambda_{0}}} B_{X}(x, l)$. Then $U \subset U_{V_{\lambda}}$ for every $\lambda \in I_{\lambda_{0}}$, and all assumptions of Theorem 2.3 are satisfied with $V:=U$ and $X:=X_{a}^{b}, a:=\max \left\{\lambda_{0}-l, 0\right\}, b:=$ $\min \left\{\lambda_{0}+l, 1\right\}$. Hence, for every $\lambda \in I_{\lambda_{0}}$,

$$
\operatorname{ind}_{K}\left(\pi_{\lambda}, I_{\lambda}, V_{\lambda}\right)=\operatorname{ind}\left(g_{\lambda}, U_{V_{\lambda}}\right)=\operatorname{ind}\left(g_{\lambda}, U\right)=\text { const } .
$$

By the compactness of $[0,1]$ we get ind $\operatorname{in}_{K}\left(\pi_{0}, I_{0}, V_{0}\right)=\operatorname{ind}_{K}\left(\pi_{1}, I_{1}, V_{1}\right)$.

\section{Sufficient conditions for the homotopy property}

As noted in Remark 2.6, conditions which are sufficient for the homotopy property (see Theorem 2.3) need a deeper study. We start with a rather obvious observation:

Remark 3.1 If $U$ is an open subset of each $A_{i}, i \in\{1, \ldots, m\}$, then $U$ is open in $X:=$ $\bigcup_{i=1}^{m} A_{i}$.

Indeed, in a simple proof for every $x \in U$ and $i \in\{1, \ldots, m\}$ we find $r_{i}>0$ such that $U \supset B_{A_{i}}\left(x, r_{i}\right)=B_{X}\left(x, r_{i}\right) \cap A_{i}$. Taking $r:=\min \left\{r_{1}, \ldots, r_{m}\right\}$ we immediately obtain

$$
B_{X}(x, r)=\bigcup_{i=1}^{m}\left(B_{X}(x, r) \cap A_{i}\right)=\bigcup B_{A_{i}}(x, r) \subset U,
$$

which means that $U$ is open in $X$.

It is easily seen that the above arguments fail in the case of infinitely many sets $A_{i}$. 
Below we present several results which give, aside from the main aim of the section, interesting properties of exit sets.

Proposition 3.2 If $\left(f_{\lambda}\right), \lambda \in[0,1]$, is a continuous family of vector fields in $\mathbb{R}^{n}$ generating, via equations $\dot{x}=f_{\lambda}(x)$, a continuous family of semiflows $\pi_{\lambda}$, and $K$ is a closed subset of $\mathbb{R}^{n}$ with compact exit sets $K_{\lambda}^{-}$, then the map $\phi^{-}:[0,1] \multimap \mathbb{R}^{n}, \phi^{-}(\lambda)=K_{\lambda}^{-}$is lsc (lower semicontinuous).

Proof Suppose that $\phi^{-}$is not lsc. Then there are $\lambda_{0} \in[0,1]$ and $\varepsilon>0$ such that for every $\delta>0$ we can find $\lambda \in[0,1]$ with $\left|\lambda-\lambda_{0}\right|<\delta$ and $K_{\lambda_{0}}^{-} \not \subset N_{\varepsilon}\left(K_{\lambda}^{-}\right)$, where $N_{\varepsilon}(\cdot)$ denotes an $\varepsilon$-neighborhood of a set. Taking $\delta=\frac{1}{m}, m \geq 1$, we obtain, for every $m \geq 1$, a number $\lambda_{m} \in\left(\lambda_{0}-\frac{1}{m}, \lambda_{0}+\frac{1}{m}\right) \cap[0,1]$ and a point $x_{m} \in K_{\lambda_{0}}^{-}$such that $\operatorname{dist}\left(x_{m}, K_{\lambda_{m}}^{-}\right) \geq \varepsilon$.

By the compactness of $K_{\lambda_{0}}$ we obtain, up to subsequence, that $x_{m} \rightarrow x_{0}$ for some $x_{0} \in K_{\lambda_{0}}^{-}$. Consider a family of Cauchy problems

$$
\left\{\begin{array}{l}
\dot{x}=f_{\lambda_{m}}(x), \\
x(0)=x_{0}
\end{array}\right.
$$

with solutions $y_{m}$ defined on a common interval $[0, T], T>0$. From the convergence $\lambda_{m} \rightarrow \lambda_{0}$ it follows that $y_{m} \rightarrow y$, where $y$ is a solution to

$$
\left\{\begin{array}{l}
\dot{x}=f_{\lambda_{0}}(x) \\
x(0)=x_{0}
\end{array}\right.
$$

We know that, for every $\eta>0$, there is a positive time $s \in(0, \rho)$ with $y(s) \notin K$. Take $s$ such that $\left|x_{0}-y(t)\right|<\frac{\varepsilon}{3}$ for every $t \in[0, s]$. By the convergence $y_{m} \rightarrow y$ and closedness of the set $K$ we get that $y_{m}(s) \notin K$ for sufficiently big $m \geq 1$. It implies that $y_{m}\left(s_{m}\right) \in K_{\lambda_{m}}^{-}$ for some $s_{m} \in[0, s)$.

We can assume that $m$ is so big that $\left|y_{m}(t)-y(t)\right|<\frac{\varepsilon}{3}$ for every $t \in[0, s]$. Then

$$
\left|y_{m}\left(s_{m}\right)-x_{m}\right| \leq\left|y_{m}\left(s_{m}\right)-y\left(s_{m}\right)\right|+\left|y\left(s_{m}\right)-x_{0}\right|+\left|x_{0}-x_{m}\right|<\varepsilon,
$$

which contradicts the inequality $\operatorname{dist}\left(x_{m}, K_{\lambda_{m}}^{-}\right) \geq \varepsilon$.

Under some regularity assumptions on the set $K$ one obtains the following

Proposition 3.3 Assume that $K \subset \mathbb{R}^{n}$ is a closed $C^{1}$-manifold with a boundary $\partial K,\left(f_{\lambda}\right)$ is as in Proposition 3.2, and int ${ }_{\partial K} K_{\lambda}^{-}=K_{\Rightarrow}^{\lambda}$ for every $\lambda \in[0,1]$ (see Remark 2.5.4). Then the map $\phi^{+}:[0,1] \multimap \mathbb{R}^{n}, \phi^{+}(\lambda)=\operatorname{cl}\left(\partial K \backslash K_{\lambda}^{-}\right)$has a closed graph.

Proof Assume that $[0,1] \ni \lambda_{m} \rightarrow \lambda$ and $y_{m} \rightarrow y$, where $y_{m} \in \operatorname{cl}\left(\partial K \backslash K_{\lambda_{m}}^{-}\right)$. Take a sequence $\left(x_{m}\right)$ such that $x_{m} \in \partial K \backslash K_{\lambda_{m}}^{-}$and $\left|x_{m}-y_{m}\right|<\frac{1}{n}$, for every $n \geq 1$. Then, obviously, $x_{m} \rightarrow y$. We shall show that $y \in \operatorname{cl}\left(\partial K \backslash K_{\lambda_{0}}^{-}\right)$.

Suppose it is not true. Then $y \in i n t_{\partial K} K_{\lambda_{0}}^{-}$which implies, by the assumption, that $\left\langle n(y), f_{\lambda_{0}}(y)\right\rangle=: d>0$, where $n(y)$ stands for the outer normal vector to $K$ in $y$. Using the continuity of the family $\left(f_{\lambda}\right)$, the map $n(\cdot)$ and an inner product, we get, for $m$ big enough,

$$
\begin{aligned}
\left\langle n\left(x_{m}\right), f_{\lambda_{m}}\left(x_{m}\right)\right\rangle= & \left\langle n\left(x_{m}\right)-n(y), f_{\lambda_{m}}\left(x_{m}\right)\right\rangle+\left\langle u(y), f_{\lambda_{0}}(y)\right\rangle \\
& +\left\langle u(y), f_{\lambda_{m}}\left(x_{m}\right)-f_{\lambda_{0}}(y)\right\rangle \\
\geq & -\left|n\left(x_{m}\right)-n(y)\right|\left(\left|f_{\lambda_{0}}(y)\right|+1\right)+d-|n(y)|\left|f_{\lambda_{m}}\left(x_{m}\right)-f_{\lambda_{0}}(y)\right| .
\end{aligned}
$$

It implies that $\left\langle n\left(x_{m}\right), f_{\lambda_{m}}\left(x_{m}\right)\right\rangle>0$ for sufficiently big $m$. Hence, $x_{m} \in K_{\lambda_{m}}^{-}$; a contradiction. 
We use Propositions 3.2 and 3.3 to prove:

Proposition 3.4 Let assumptions of Proposition 3.3 be satisfied with compact exit sets $K_{\lambda}^{-}$. Assume that $U \subset$ int $_{\partial K} K_{\lambda_{0}}^{-}$is open in $K_{\lambda_{0}}^{-}$. Then

$$
\forall x \in U \exists r>0 \exists \delta>0 \forall \lambda \in\left(\lambda_{0}-\delta, \lambda_{0}+\delta\right) \cap[0,1]: \operatorname{dist}\left(x, \partial_{\partial K} K_{\lambda}^{-}\right)>r .
$$

Proof Suppose that for some $x \in U$ we have

$$
\forall r>0 \forall \delta>0 \exists \lambda \in\left(\lambda_{0}-\delta, \lambda_{0}+\delta\right) \cap[0,1]: \operatorname{dist}\left(x, \partial_{\partial K} K_{\lambda}^{-}\right) \leq r .
$$

Take $r=\frac{1}{m}$ and $\delta=\frac{1}{m}$. Then, for every $m \geq 1$, we can find $\lambda_{m} \in\left(\lambda_{0}-\frac{1}{m}, \lambda_{0}+\frac{1}{m}\right) \cap$ $[0,1]$ such that $\operatorname{dist}\left(x, \partial_{\partial K} K_{\lambda_{m}}^{-}\right) \leq \frac{1}{m}$ which implies that there exists $x_{m} \in \partial_{\partial K} K_{\lambda_{m}}^{-}$with $\left|x-x_{m}\right| \leq \frac{1}{m}$.

From Proposition 3.2 it follows that for every $m \geq 1$ there exists $\eta>0$ such that $K_{\lambda_{0}}^{-} \subset N_{\frac{1}{m}}\left(K_{\lambda}^{-}\right)$for each $\lambda \in[0,1]$ with $\left|\lambda-\lambda_{0}\right|<\eta$. Therefore, we can assume that $K_{\lambda_{0}}^{-} \subset N_{\frac{1}{m}}\left(K_{\lambda_{m}}^{-}\right)$. By Proposition 3.3 the map $\phi^{+}:[0,1] \multimap \mathbb{R}^{n}, \phi^{+}(\lambda)=\operatorname{cl}\left(\partial K \backslash K_{\lambda}^{-}\right)$has a closed graph. Hence, since $\lambda_{m} \rightarrow \lambda_{0}, x_{m} \rightarrow x$ and $x_{m} \in \partial_{\partial K} K_{\lambda_{m}}^{-} \subset \operatorname{cl}\left(\partial K \backslash K_{\lambda_{m}}^{-}\right)$, we obtain that $x \in \operatorname{cl}\left(\partial K \backslash K_{\lambda_{0}}^{-}\right)$. But we know that $x \in K_{\lambda_{0}}^{-}$, so $x \in \partial_{\partial K} K_{\lambda_{0}}^{-}$which contradicts the assumption that $x \in U \subset i n t_{\partial K} K_{\lambda_{0}}^{-}$.

Assumptions of Proposition 3.4 are sufficient for one of the main proof arguments in the homotopy property of the impulsive index (see Remark 2.6) as we can see in the following:

Proposition 3.5 Under assumptions of Proposition 3.4 every set $U \subset$ int $_{\partial K} K_{\lambda_{0}}^{-}$, which is open in $K_{\lambda_{0}}^{-}$and contained in each $K_{\lambda}^{-}$, is also open in $X=\bigcup_{\lambda \in[0,1]} K_{\lambda}^{-}$.

Proof It is easy to see that $U$ is open in $\partial K$. Moreover, $U \subset X \subset \partial K$. This completes the proof.

Open problem. Is the following property true?

If $\left(f_{\lambda}\right), \lambda \in[0,1]$, is a continuous family of vector fields in $\mathbb{R}^{n}$ generating a continuous family of semiflows $\pi_{\lambda}$, and $K$ is a closed subset of $\mathbb{R}^{n}$ with compact exit sets $K_{\lambda}^{-}$, then the $\operatorname{map} \phi^{+}:[0,1] \multimap \mathbb{R}^{n}, \phi^{+}(\lambda)=\operatorname{cl}\left(\partial K \backslash K_{\lambda}^{-}\right)$has a closed graph.

In other words, is Proposition 3.3 true without $C^{1}$-regularity of the set and an additional assumption $i n t_{\partial K} K_{\lambda}^{-}=K_{\Rightarrow}^{\lambda}$ on the exit set?

\section{Computation of the index via Poincaré-type maps}

In the section we briefly remind the technique of computation of the fixed point index $\operatorname{ind}\left(g_{\lambda}, U\right)$ and, consequently, the impulsive index $\operatorname{ind}_{K}(\pi, I, V)$, proposed firstly in [14]. The idea is partially based on the Poincaré map and Poincaré section technique used in the study of periodic trajectories of ordinary smooth flows.

Let $K$ be a closed subset of $\mathbb{R}^{n}$, and we are given a problem (1.1) with the differential equation generating a closed exit set $K^{-}$which is a strong deformation retract of $K$ via the homotopy along trajectories (see Sect. 2). Let $M \subset K^{-}$be a barrier, and $V \subset K^{-}$be any subset open in $K^{-}$. 
In what follows we assume that the vector field $f: \mathbb{R}^{n} \rightarrow \mathbb{R}^{n}$ and the impulse function $I: M \rightarrow \mathbb{R}^{n}$ are of class $C^{1}$, at least. Let $p \in M \cap V$ be a fixed point of $g=r I: M^{I} \rightarrow K^{-}$. Denote $q:=I(p) \in K$ and the time $T:=\tau_{K}(q)$. Obviously, $p=\pi(q, T)$, where $\pi$ is a flow generated by $f$. We shall also use the assumption that $M$ is a level set $\left\{x \in \mathbb{R}^{n} \mid \gamma(x)=0\right\}$ for some smooth function $\gamma: \mathbb{R}^{n} \rightarrow \mathbb{R}$.

Finally assume that $p \in \int_{\partial K}(M \cap V)$, the vector $f(p)$ is transversal to $\partial K$, and $f(q)$ is transversal to $I(M)$. Then, for some $N \subset M \cap V$ with $p \in i n t_{K^{-}} N$ there exists a $\delta>0$ such that for every $y \in\{\pi(x, t)|x \in N| t \mid,<\delta\}$ one can find a unique $\delta(y) \in(-\delta, \delta)$ with $\pi(y, \delta(y)) \in N$. Such a neighborhood $N$ is a Poincaré section for $\pi$.

Under the above assumptions we can check that the exit time function $\tau_{K}$ is smooth in a neighborhood of $q$. Without any loss of generality we can assume that $I(N)$ is this neighborhood. Since $g(x)=\pi\left(I(x), \tau_{K}(I(x))\right)$, we get

$$
g_{x}(x)=\pi_{x}\left(I(x), \tau_{K}(I(x))\right) I_{x}(x)+\pi_{t}\left(I(x), \tau_{K}(I(x))\right)\left(\tau_{K}\right)_{x}(I(x)) I_{x}(x),
$$

where, e.g., $g_{x}(x)$ stands for the state variable derivative of $g$. Remind that (one easily checks it) $t \mapsto f(\pi(q, t))$ is a solution on $[0, T]$ to the linear differential equation $\dot{y}=f_{x}(\pi(q, t)) y$ with an initial condition $y(0)=f(q)$. It is seen that $y(T)=f(p)$. We know also that $t \mapsto \pi_{x}(q, t)$ is a fundamental matrix solution to the equation $\dot{Y}=f_{x}(\pi(q, t)) Y$ with $Y(0)=\pi_{x}(q, 0)$. Hence, we get

$$
Y(T) f(q)=\pi_{x}(q, T) f(q)=f(\pi(q, T))=f(p) .
$$

Now we choose in $\mathbb{R}^{n}$ two bases $\mathcal{B}_{1}:=\left\{f(p), a_{2}, \ldots, a_{n}\right\}$ and $\mathcal{B}_{2}:=\left\{f(q), b_{2}, \ldots, b_{n}\right\}$ such that $a_{i}$ are parallel to $M$ at $p$ (they form a basis in a tangent space to $M$ at $p$ ) and $b_{i}$ are parallel to $I(M)$ at $q$. We consider a linear map $\pi_{x}(q, T)$ in bases $\mathcal{B}_{2}$ and $\mathcal{B}_{1}$ obtaining the matrix

$$
\pi_{x}(q, T)=\left[\begin{array}{ll}
1 & A \\
0 & B
\end{array}\right], \text { where } A \in M_{1 \times(n-1)} \text { and } B \in M_{(n-1) \times(n-1)} .
$$

If $w \in \mathbb{R}^{n}$ is parallel to $M$ at $p$, then it has the form $w=\left[\begin{array}{c}0 \\ w_{M}\end{array}\right]$. Moreover, $I_{x}(p)\left[\begin{array}{c}0 \\ w_{M}\end{array}\right]=$ $\left[\begin{array}{c}0 \\ w_{I M}\end{array}\right]$, where $\left[\begin{array}{c}0 \\ w_{I M}\end{array}\right]$ is a vector parallel to $I(M)$ at $q$.

Now, using (4.1) we can compute a derivative of a Poincaré map. Let $w$ be a vector parallel to $M$ at $p$. Then

$$
\begin{aligned}
D g(p) w & =\pi_{x}(q, T) I_{x}(p)\left[\begin{array}{c}
0 \\
w_{M}
\end{array}\right]+\pi_{t}(q, T)\left(\tau_{K}\right)_{x}(q) I_{x}(p)\left[\begin{array}{c}
0 \\
w_{M}
\end{array}\right] \\
& =\pi_{x}(q, T)\left[\begin{array}{c}
0 \\
w_{I M}
\end{array}\right]+f(p)\left(\tau_{K}\right)_{x}(q)\left[\begin{array}{c}
0 \\
w_{I M}
\end{array}\right] \\
& =\left[\begin{array}{l}
A w_{I M} \\
B w_{I M}
\end{array}\right]+\left[\begin{array}{l}
c \\
0
\end{array}\right],
\end{aligned}
$$

for some $c \in \mathbb{R}$. Since $g$ maps $M$ into $K^{-}(f)$, we obtain in the basis $\mathcal{B}_{1}$

$$
D g(p) w=\left[\begin{array}{c}
0 \\
B w_{I M}
\end{array}\right]=\left[\begin{array}{cc}
0 & 0 \\
0 & B \cdot T_{p} I
\end{array}\right] w,
$$

where $T_{p} I: T_{p} M \rightarrow T_{q} I(M)$ is the derivative of $I$ treated as a map between manifolds. This is a very good information because in a computation of the degree one uses eigenvalues 


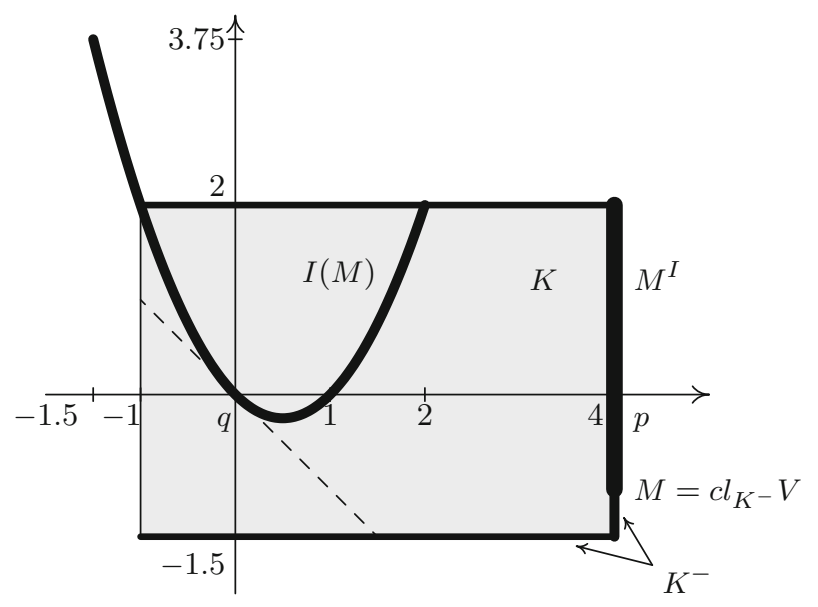

Fig. 3 Illustration to Example 4.1

of the derivative. Here, eigenvalues of $D g(p)$ correspond to those of the matrix $B \cdot T_{p} I \in$ $M_{(n-1) \times(n-1)}$.

Taking a sufficiently small neighborhood $U$ of $p$ we obtain

$$
\operatorname{ind}(g, p):=\operatorname{ind}(g, U)=\operatorname{deg}(i-g, U)=(-1)^{\beta},
$$

where $\beta$ is the sum of the multiplicities of the negative real eigenvalues of $\operatorname{id}_{T_{p} M}-B \cdot T_{p} I$.

To illustrate how the above technique works in practice we present an example which is a modification of Example 4.1 in [14].

Example 4.1 We have a system of equations

$$
\left\{\begin{array}{l}
\dot{x}=1 \\
\dot{y}=y
\end{array}\right.
$$

generating a global flow $\pi((x, y), t)=\left(x+t, y e^{t}\right)$ on $\mathbb{R}^{2}$. For the set of constraints $K:=$ $[-1,4] \times[-3 / 2,2]$ we obtain the exit set $K^{-}=K_{1} \cup K_{2} \cup K_{3}$, where $K_{i}$ are the faces $x=4, y=2$ and $y=-3 / 2$ of $K$, respectively (see Fig. 3). Let $M:=\{(4, y) \mid y \in$ $[-3 / 2,2]\}=c l_{K^{-}}\{(4, y) \mid y \in(-3 / 2,2)\}$ and $I: M \rightarrow \mathbb{R}^{n}$ be an impulse function given by $I(x, y):=(y, y(y-1))$. For simplicity, take $V=\{(4, y) \mid y \in(-3 / 2,2)\}$ which is open in $K^{-}$.

It is easy to check that $M^{I}=I^{-1}(I(M) \cap K)=\{(4, y) \mid y \in[-1,2]\}$ and $U_{V}=$ int $_{K^{-}}\left(I^{-1}(I(V \cap M) \cap K) \cap V\right)=\{(4, y) \mid y \in(-1,2)\}$.

Since $g(4,-1)=I(4,-1)=(-1,2) \in K^{-}$and $g(4,2)=I(4,2)=(2,2) \in K^{-}$, we have ind $\left(g, U_{V}\right)=0$. On the other hand, it is evident that $p:=(4,0)$ is a fixed point of $g$ with $q:=I(p)=(0,0)$. Now, as in [14], we take two bases $\mathcal{B}_{1}:=\{(1,0),(0,1)\}$ and $\mathcal{B}_{2}:=\{(1,0),(1,-1)\}$ of $\mathbb{R}^{2}$, and notice that $\pi_{x}(q, T)=\left[\begin{array}{cc}1 & 1 \\ 0 & -e^{T}\end{array}\right]$ in bases $\mathcal{B}_{2}$ and $\mathcal{B}_{1}$, i.e., $\pi_{x}(q, T):\left(\mathbb{R}^{2}, \mathcal{B}_{2}\right) \rightarrow\left(\mathbb{R}^{2}, \mathcal{B}_{1}\right)$, where $T=\tau_{K}(q)=4$. 
Since $D I(p)=\left[\begin{array}{ll}0 & 0 \\ 0 & 1\end{array}\right]$ in bases $\mathcal{B}_{1}$ and $\mathcal{B}_{2}$, for any vector $w=\left[\begin{array}{l}0 \\ v\end{array}\right]$ parallel to $M$ we get

$$
\operatorname{Dg}(p)(w)=\operatorname{Dg}(p)\left[\begin{array}{l}
0 \\
v
\end{array}\right]=\left[\begin{array}{l}
0 \\
-e^{T} v
\end{array}\right]=\left[\begin{array}{ll}
0 & 0 \\
0 & -e^{T}
\end{array}\right]\left[\begin{array}{l}
0 \\
v
\end{array}\right] .
$$

This implies that $\operatorname{ind}(g, p):=\operatorname{ind}(g, W)=\operatorname{deg}(i-g, W)=\operatorname{sgn}\left(1+e^{T}\right)=1$ for some small neighborhood $W$ of $p$ in $U_{V}$. From the additivity property of the fixed point index we obtain ind $\left(g, U_{V} \backslash c l_{K^{-}(f)} W\right)=\operatorname{ind}\left(g, U_{V}\right)-\operatorname{ind}(g, W)=-1 \neq 0$ which implies the existence of another periodic trajectory.

\section{Concluding remarks}

We start the section with a discussion on a possible alternative definition of the impulsive index. Instead of conditions (A1)-(A4) from Sect. 2 we assume

(Z1) $\partial K$ is a closed ANR, and the exit set $K^{-}$is closed,

(Z2) $M=c l_{\partial K}\left(\right.$ int $\left._{\partial K} M\right)$,

(Z3) $I$ is a compact map, i.e. $I(M)$ is relatively compact,

(Z4) $\operatorname{dist}(I(M), M)>0$.

As before, we assume (2.1) with the retraction $r: K \rightarrow K^{-}, r(x)=\pi\left(x, \tau_{K}(x)\right)$. Define $M^{I}:=I^{-1}(K)$ and $g: M^{I} \rightarrow \partial K, g(x):=r(I(x))$ for $x \in M^{I}$. Notice that a codomain is different from the one in (2.3). Of course, $g\left(M^{I}\right) \subset K^{-}$.

Let $V$ be an arbitrary open subset of $\partial K$. We define

$$
U_{V}:=\operatorname{int}_{\partial K}\left(I^{-1}(K) \cap V\right) \subset V,
$$

and assume that

$$
\text { Fix } g \cap U_{V} \text { is a compact set. }
$$

Now we put

$$
\operatorname{ind}_{K}^{\prime}(\pi, I, V):=\operatorname{ind}\left(g, U_{V}\right),
$$

where ind $\left(g, U_{V}\right)$ is a fixed point index in an ANR $\partial K$, not in $K^{-}$.

This index has standard properties (see Sect. 2) with the homotopy property under easier assumptions. We give, for instance, an analogue to Theorem 2.3 as the following

Theorem 5.1 (Homotopy I) Let $\pi_{\lambda}, \lambda \in[0,1]$, be a continuous family of semiflows. Assume that, for every $\lambda \in[0,1]$, the exit set $K_{\lambda}^{-}$for $\pi_{\lambda}$ is closed, there are retractions $r_{\lambda}: K \rightarrow K_{\lambda}^{-}, r_{\lambda}(x)=\pi_{\lambda}\left(x, \tau_{K}(x)\right)$, and $M=c_{\partial K}\left(\right.$ int $\left._{\partial K} M\right)$. Moreover, assume that the set $\bigcup_{\lambda \in[0,1]} r_{\lambda}(I(M) \cap K)$ is relatively compact.

Let $V$ be an open subset of $\partial K$, and Fix $g_{\lambda} \cap U_{V_{\lambda}}$ is compact.

Then the numbers ind ${ }_{K}^{\prime}\left(\pi_{\lambda}, I, V\right)$ are well defined, and

$$
\operatorname{ind}_{K}^{\prime}\left(\pi_{0}, I, V\right)=\operatorname{ind}_{K}^{\prime}\left(\pi_{1}, I, V\right) .
$$

Proof Notice that $U_{V_{\lambda}}=$ int $_{\partial K}\left(I^{-1}(K) \cap V\right)=U_{V_{0}}$ is independent of the choice of $\lambda$. So, we have a homotopy $g_{\lambda}: U_{V_{0}} \rightarrow \partial K$ with assumptions allowing to apply the homotopy property of the index on ANRs. This finishes the proof. 
Fig. $4 \operatorname{ind}_{K}(\pi, I, V)=1$ for $V=M=K^{-}$

Fig. $5 \operatorname{ind}_{K}^{\prime}\left(\pi, I, V_{0}\right)$ is not defined
Fig. $6 \operatorname{ind}_{K}\left(\pi, I, V_{1}\right)=$ $\operatorname{ind}_{K}^{\prime}\left(\pi, I, V_{1}\right)=-1$ and $\operatorname{ind}_{K}(\pi, I, V)=1$
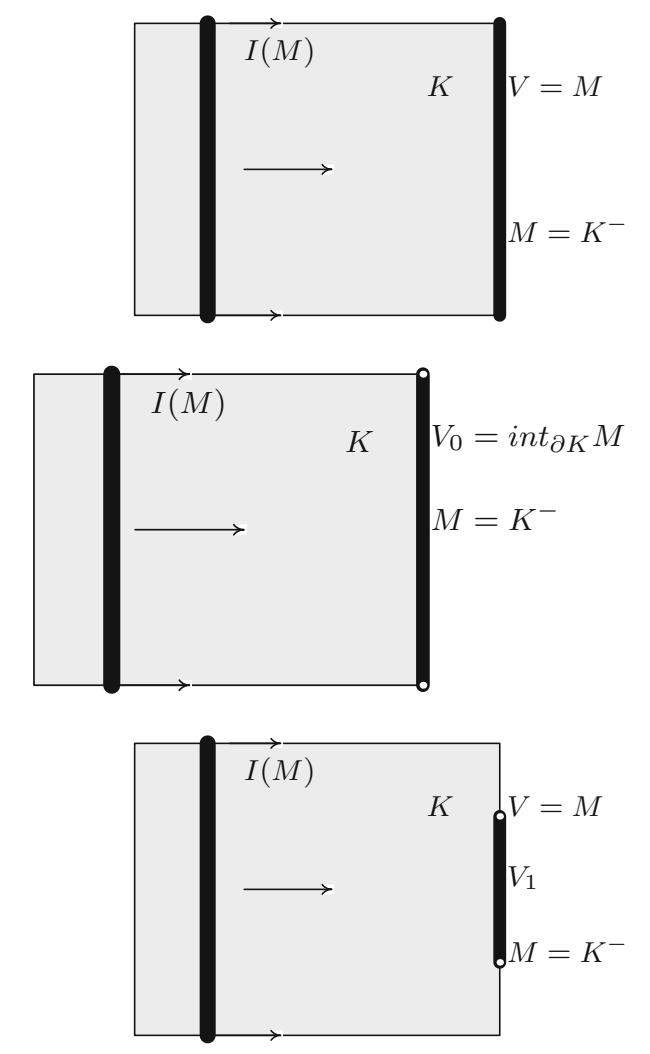

One would think that the index ind $_{K}^{\prime}$ is better because it simplifies considerations. However, it does not cover some important cases. Indeed, the set $V$ cannot be equal to $K^{-}$since $K^{-}$ is closed and usually not open in $\partial K$. The following concrete example shows the difference between the results obtained by the use of ind ${ }_{K}$ and ind $_{K}^{\prime}$.

Example 5.2 Let $K$ be a rectangle in $\mathbb{R}^{2}$, for instance $K=[0,3] \times[0,2]$ and $\pi$ is generated by the equation $(\dot{x}, \dot{y})=(g(t), 0)$ with $g(t)>0$. Then, obviously, $K^{-}=\{3\} \times[0,2]$. Put $M=K^{-}$, see Figs. 4, 5, 6 and 7 .

At first, assume that $I(x, y):=(1, y)$ and $V=M=K^{-}$which is allowed in Sect. 2 (Fig. 4). Then $U_{V}=K^{-}$and $\operatorname{ind}_{K}(\pi, I, V)=\operatorname{ind}\left(i d, K^{-}\right)=\chi\left(K^{-}\right)=1$, where $\chi\left(K^{-}\right)$ is the Euler characteristic of the set $K^{-}$(equal to the Lefschetz number of $i d$ ).

Notice that, for every open subset $V_{0}$ of $\partial K$, the set $U_{V_{0}}$ constructed in (5.1), even the largest one, is not equal to $K^{-}$. It implies that the index $\operatorname{ind}_{K}^{\prime}\left(\pi, I, V_{0}\right)$ for no $V_{0}$ (see Fig. 5) is well defined, because Fix $i d \cap U_{V_{0}}=U_{V_{0}}$ is not compact.

Now, let $M, V, V_{0}$ be as above, and $I(x, y):=(1, \sqrt[3]{y-1}+1)$. Consider $V_{1}:=\{3\} \times$ $(1 / 2,3 / 2)$ (see Figs. 6, 7). Since $U_{V_{1}}=V_{1}$ in both approaches [see (2.4) and (5.1)], and $g(3, y)=(3, \sqrt[3]{y-1}+1)$, we easily obtain $\operatorname{ind}_{K}\left(\pi, I, V_{1}\right)=\operatorname{ind}_{K}^{\prime}\left(\pi, I, V_{1}\right)=-1$ and $\operatorname{ind}_{K}(\pi, I, V)=1$, while $\operatorname{ind}_{K}^{\prime}\left(\pi, I, V_{0}\right)=-1$.

Our final remarks concerns possible generalizations of the impulsive index. One knows that a differential inclusion $\dot{x} \in F(x)$ taken instead of $\dot{x}=f(x)$ in (1.1), where $F$ is upper semicontinuous (usc) convex compact valued with a sublinear growth, generates a 
Fig. $7 \operatorname{ind}_{K}\left(\pi, I, V_{0}\right)=$ $\operatorname{ind}_{K}^{\prime}\left(\pi, I, V_{0}\right)=-1$

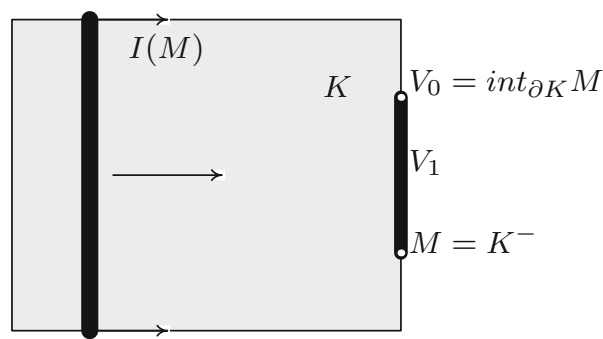

multivalued dynamical system. Moreover, the solution map $\mathbb{R}^{n} \ni x_{0} \multimap S_{F}\left(x_{0}\right)$, where $S_{F}\left(x_{0}\right) \in C\left([0, \infty), \mathbb{R}^{n}\right)$ is a solution set for the Cauchy problem with $x(0)=x_{0}$, is usc with compact $R_{\delta}$-values. Under suitable assumptions on the set of constraints $K$ and an exit set, one can obtain results on the existence of viable and stationary trajectories (see, e.g., $[12,13])$. If we suppose that there is no viable solution in $K$, it is sensible to assume that there exists a multivalued retraction $\Phi$ of $K$ onto $K_{e}(F)$, where

$$
K_{e}(F):=\left\{x_{0} \in \partial K \mid \exists x \in S_{F}\left(x_{0}\right): x \text { leaves } K \text { immediately }\right\}
$$

is a bigger exit set (see [14], or [12] where a discussion on two exit sets $K^{-}(F)$ and $K_{e}(F)$ is presented). Now, if $I: M \multimap \mathbb{R}^{n}$ is a multivalued impulse map, then the construction of the fixed point index of the composition $\Phi \circ I$ is still possible under suitable geometric assumptions on $K_{e}(F)$ and regularity assumptions on $I$. In particular, if $K_{e}(F)$ is a compact ANR and $I$ is usc with $R_{\delta}$-values, then the index proposed in [2] could be used (see also [15] for other versions of the index). Details of the construction and properties of the impulsive index for multivalued flows and jumps we leave for further considerations.

Acknowledgments The author is supported by the Polish NCN grant no.2013/09/B/ST1/01963. The author is indebted to the referee for her/his valuable comments and suggestions which helped to simplify notations and some proofs.

Open Access This article is distributed under the terms of the Creative Commons Attribution License which permits any use, distribution, and reproduction in any medium, provided the original author(s) and the source are credited.

\section{References}

1. Azevedo, K.A.G., Bonotto, E.M.: On asymptotic stability in impulsive semidynamical systems. J. Dyn. Control Syst. 19(3), 359-380 (2013)

2. Bader, R., Kryszewski, W.: Fixed-point index for compositions of set-valued maps with proximally $\infty$ connected values on arbitrary ANR's. Set Valued Anal. 2(3), 459-480 (1994)

3. Bainov, D., Simeonov, P.: Impulsive differential equations: periodic solutions and applications. Pitman Monographs and Surveys in Pure and Applied Mathematics, 66. Longman Scientific \& Technical, Harlow; copublished in the United States with John Wiley \& Sons Inc, New York (1993)

4. Becker, J.C., Gottlieb, D.H.: Vector fields and transfers. Manuscripta Math. 72(2), 111-130 (1991)

5. Bonotto, E.M., Ferreira, J.C.: Uniform attractors of discontinuous semidynamical systems. Collect. Math. 65(1), 47-59 (2014)

6. Brézis, H.: On a characterization of flow-invariant sets. Commun. Pure Appl. Math. 23, 261-263 (1970)

7. Cardaliaguet, P., Gabor, G., Quincampoix, M.: Equilibria and strict equilibria of multivalued maps on noninvariant sets. Ann. Polon. Math. 82, 19-37 (2003)

8. Cardinali, T., Servadei, R.: Periodic solutions of nonlinear impulsive differential inclusions with constraints. Proc. Am. Math. Soc. 132(8), 2339-2349 (2004) 
9. Cardinali, T., Servadei, R.: On the existence of solutions for nonlinear impulsive periodic viable problems. Cent. Eur. J. Math. 2(4), 573-583 (2004)

10. Ciesielski, K.: On semicontinuity in impulsive dynamical systems. Bull. Pol. Acad. Sci. Math. 52(1), 71-80 (2004)

11. Conley, C.: A new statement of Ważewski's theorem and an example. Ordin. Part. Diff. Equ. In: Proc. 4th Conf., Dundee 1976, Lect. Notes Math, vol. 564, pp. 61-71. (1976)

12. Gabor, G., Quincampoix, M.: On existence of solutions to differential equations or inclusions remaining in a prescribed closed subset of a finite-dimensional space. J. Differ. Equ. 185(2), 483-512 (2002)

13. Gabor, G.: Homotopy index for multivalued flows on sleek sets. Set Valued Anal. 13(2), 125-149 (2005)

14. Gabor, G.: The existence of viable trajectories in state-dependent impulsive systems. Nonlinear Anal. 72, 3828-3836 (2010)

15. Górniewicz, L.: Topological Fixed Point Theory of Multivalued Mappings. Springer, Dordrecht (2006)

16. Granas, A., Dugundji, J.: Fixed Point Theory. Springer Monographs in Mathematics. Springer, New York (2003)

17. Kryszewski, W., Plaskacz, S.: Periodic solutions to impulsive differential inclusions with constraints. Nonlinear Anal. 65(9), 1794-1804 (2006)

18. Mil'man, V.D., Myškis, A.D.: On the stability of motion in the presence of impulses. (Russian) Sibirsk. Mat. Ž. 1, 233-237 (1960)

19. Nagumo, M.: Über die Lage der Integralkurven gewühnlicher Differentialgleichungen. Proc. Phys.-Math. Soc. Japan, III. Ser., vol. 24, pp. 551-559. (1942)

20. Ważewski, T.: Sur un principe topologique de l'examen de l'allure asymptotique des intégrales des équations différentielles ordinaires. Ann. Soc. Polon. Math. 20, 279-313 (1947) 